\title{
Early response to the treatment of choroidal neovascularization complicating central serous chorioretinopathy: a OCT-angiography study
}

\author{
Riccardo Sacconi $\mathbb{B}^{1,2} \cdot$ Livia Tomasso $^{1}$ - Eleonora Corbelli ${ }^{1}$. Adriano Carnevali ${ }^{1,3}$ - Lea Querques ${ }^{1}$ - Stefano Casati ${ }^{2}$. \\ Francesco Bandello ${ }^{1}$ Giuseppe Querques ${ }^{1}$
}

Received: 19 November 2018 / Revised: 2 April 2019 / Accepted: 6 April 2019 / Published online: 2 July 2019

(c) The Author(s), under exclusive licence to The Royal College of Ophthalmologists 2019

\begin{abstract}
Purpose To analyze the quantitative and qualitative early changes of choroidal neovascularization (CNV) associated with chronic central serous chorioretinopathy (CSC) after treatment using optical coherence tomography-angiography (OCT-A). Methods Charts of consecutive patients with diagnosis of chronic CSC complicated by CNV were retrospectively reviewed. Included patients were divided in photodynamic therapy (PDT) or aflibercept group on the basis of the treatment received (half-fluence PDT or aflibercept $2.0 \mathrm{mg} / 0.05 \mathrm{ml}$ intravitreal injection). Main outcome measures included the changes between baseline and 1-month follow-up in CNV vessel density (VD) and area on OCT-A images after thresholding and binarization.

Results A total of 30 eyes of 26 Caucasian patients were included: 17 eyes of 15 patients in PDT group (mean age $53 \pm 11$ years) and 13 eyes of 11 patients in aflibercept group (mean age $58 \pm 8$ years $[p=0.196]$ ). In both PDT and aflibercept groups, best-corrected visual acuity improved at 1 month, and central macular thickness and subretinal fluid significantly decreased. VD did not change after the treatment in both groups $(p=0.502$ and $p=0.086)$ although $\mathrm{CNV}$ area decreased significantly (from $0.586 \pm 0.449 \mathrm{~mm}^{2}$ to $0.553 \pm 0.453 \mathrm{~mm}^{2}$ [0.041]) in the PDT group, and nonsignificantly (from $0.767 \pm$ $0.466 \mathrm{~mm}^{2}$ to $\left.0.733 \pm 0.472 \mathrm{~mm}^{2}[p=0.095]\right)$ in the aflibercept group. The same results were confirmed in the subanalysis of the 18 treatment-naïve eyes.

Conclusions We demonstrated that, despite all patients showed a favorable clinical response, VD of CNVs complicating chronic CSC did not change after treatment. These findings support the idea that arteriogenesis is the main driving force of $\mathrm{CNV}$ in pachychoroid-related macular disorders.
\end{abstract}

\section{Introduction}

Central serous chorioretinopathy (CSC) is a common disease included in the pachychoroid-related macular disorders, a spectrum of diseases with similar features including thickened choroid and dilated outer choroidal

Giuseppe Querques

giuseppe.querques@hotmail.it

1 Department of Ophthalmology, University Vita-Salute, IRCCS Ospedale San Raffaele, Milan, Italy

2 Eye Clinic, Department of Neurological, Biomedical and Movement Sciences, University of Verona, Verona, Italy

3 Department of Ophthalmology, University of "Magna Graecia", Catanzaro, Italy vessels [1]. CSC typically affects young adult men with an incidence of $\sim 0.5-1 / 10000[2,3]$.

Acute disease is characterized by a serious retinal detachment located in the macular region and it has typically a favorable course with a spontaneous regression in the first months [3]. Nevertheless, in 5-10\% of patients the acute episodes may not resolve and progress to a chronic form $[4,5]$, characterized by persistent serous retinal detachment associated with diffuse retinal pigment epithelium (RPE) alterations, cystoid retinal degeneration or pigment epithelium detachment, and, in some cases, choroidal neovascularization (CNV) [4-6].

$\mathrm{CNV}$ in chronic CSC is included in the group of pachychoroid neovasculopathy, which is defined as type 1 neovascularization associated with choroidal thickening in absence of characteristic age-related macular degeneration (AMD) or degenerative changes [7]. This pattern of CNV 
has different imaging features in comparison to type 1 neovascularization in AMD; in particular, choroidal vascular plaque in the late phases of indocyanine green angiography (ICGA) is displayable in only $25-42 \%$ of cases [8-11].

Optical coherence tomography angiography (OCT-A) is a noninvasive tool that employs amplitude or phase decorrelation to detect motion and has recently been introduced into clinical practice. This system has provided insight into the pathophysiological mechanisms of several retinal diseases [12-18]. Recently, OCT-A demonstrated greater sensitivity and specificity in comparison with conventional dye angiographies in detecting type $1 \mathrm{CNV}$ in pachychoroid-related macular disorders [16-18]. Nevertheless, no studies analyzed the quantitative and qualitative features of CNV in chronic CSC using OCT-A, and, in particular, CNV modifications after treatment are still largely unknown. Therefore, we conducted this study to evaluate the quantitative and qualitative features of $\mathrm{CNV}$ associated with chronic CSC before and early after treatment.

\section{Methods}

In this retrospective cohort study, consecutive patients with diagnosis of chronic CSC complicated by CNV were collected from the Medical Retina and Imaging Unit of San Raffaele Hospital (Milan, Italy) between January 2016 and August 2017. The study adhered to the 1964 Helsinki declaration and its later amendments. Informed consent was obtained from all individual participants included and it was approved by the Local Institutional Review Board.

Inclusion criteria were: (1) patients aged more than 18 years, (2) diagnosis of chronic CSC (defined as history of no spontaneous regression for at least 6 months and/or with other past episodes confirmed by previous structural optical coherence tomography [OCT]), (3) development of $\mathrm{CNV}$ lesion during the CSC history confirmed on the basis of OCT-A. Furthermore, all eyes had to show the presence of at least one pachychoroid feature, defined as any of the following: (1) choroidal thickness more than $270 \mu \mathrm{m}$, (2) the presence of pachyvessels, (3) history of previous episodes of CSC [8].

Exclusion criteria were: (1) history of other retinal disorders (including AMD, diabetic retinopathy, uveitis or pathologic myopia), (2) presence of any other systemic condition that contraindicated treatment of $\mathrm{CNV}$, (3) relevant opacities of the optic media and low quality images, (4) previous photodynamic therapy (PDT) and/or antivascular endothelial growth factor (VEGF) intravitreal injection in the last 6 months. Patients that received PDT and/or antiVEFG intravitreal injections before 6 months from the inclusion were considered "previously treated" patients, otherwise the patients were considered "treatment-naïve".

All included patients were divided in two different groups on the basis of the treatment received: the PDT group (treated with half-fluence PDT guided by ICGA between January 2016 and October 2016), and the aflibercept group (treated with 1 aflibercept $2.0 \mathrm{mg} / 0.05 \mathrm{ml}$ intravitreal injection between November 2016 and August 2017).

At baseline, each patient underwent a complete ophthalmologic examination, including BCVA on ETDRS chart and converted into logarithm of the minimal angle of resolution (LogMar) for statistical analysis, infrared reflectance (IR), fundus autofluorescence (FAF), structural spectral domain OCT (SD-OCT), and OCT-A. IR, FAF and structural SD-OCT were obtained using Spectralis Heidelberg Retinal Angiograph + OCT (Heidelberg Engineering, Heidelberg, Germany), whereas OCT-A was obtained using AngioPlex OCT angiographic imaging installed on the high definition OCT CIRRUSTM Model 5000 (Carl Zeiss Meditec, Dublin, CA, USA).

Patients included (17 eyes of 15 patients in the PDT group and 13 eyes of 11 patients in the aflibercept group) were followed after treatment and revaluated at 1 month with BCVA, SD-OCT, and OCT-A examinations.

In order to achieve a better visualization of the choroid, enhanced depth imaging OCT was used in all acquisitions. Central macular thickness (CMT) was assessed in the central 1-mm-diameter circle of ETDRS thickness map using the Spectralis Software (Heidelberg Eye Explorer 1.9.11.0, Heidelberg, Germany). Subretinal fluid (SRF) was manually measured as the distance between the end of the outer segment and the RPE at the point of maximum amplitude. Subfoveal choroidal thickness (SFCT) was manually evaluated as the distance between the Bruch's membrane interface and on the sclerochoroidal interface under the fovea. All measurements on structural cross-sectional OCT were collected by two expert retina specialists (EC and AC).

Main outcome measures included the changes between baseline and 1-month follow-up in CNV vessel density (VD) and area on OCT-A images after thresholding and binarization.

\section{OCT-A images acquisition and analysis}

In all patients, we performed a $3 \times 3 \mathrm{~mm}$ scanning area including the CNV. All acquisitions were performed using FastTrac $^{\mathrm{TM}}$ retinal-tracking technology to reduce motion artifacts and the minimum strength of OCT-A images was 7 out of 10 . Regarding the evaluation of $\mathrm{CNV}$ features, projection artefacts were removed using the proprietary software in order to better identify the CNV. The automatic RPE-RPE fit segmentation provided by the OCT-A 
software was manually adjusted by two expert retina specialists (E.C. and A.C.) for correct visualization and to optimize the appearance of the $\mathrm{CNV}$, at baseline and at 1-month follow-up. Disagreement on the interpretation of the different features was resolved by a senior expert retina specialist (G.Q.).

All images were exported into ImageJ 1.50 (National Institutes of Health, Bethesda, Maryland, USA) software. The CNV area was manually outlined using the polygon selection tool in the selected slab, and its dimension expressed as squared millimeters $\left(\mathrm{mm}^{2}\right)$ was calculated. The $\mathrm{CNV}$ area was measured by two independent readers (EC and $\mathrm{AC}$ ) and the mean measurement was used for the analysis. VD was calculated using Otsu's thresholding to binarize each image $[13,14]$. All area outside the CNV was colored to pure blue. White pixels were considered as vessel, black pixels as background and VD was calculated as the ratio between the white pixel and the total pixels after pure blue exclusion.

\section{Statistical analysis}

The data obtained were analyzed using SPSS Statistics Version 20 (IBM, Armonk, New York, USA). Results of descriptive analyses were expressed as mean \pm standard deviation for quantitative variables, and as count and percentage for categorical variables. Intraclass correlation coefficient (ICC; 95\% confidence intervals [CI]) was used to test the interobserver reproducibility between the two readers in CNV area measurements. Fisher's exact test was used to test the association between categorical variables. Kolmogorov-Smirnov test was used to verify the Gaussian distribution of continuous variables. Comparisons of age, BCVA, CMT, SRF, and SFCT between PDT group and aflibercept group were performed using the Student independent samples $t$-test. Comparisons of BCVA, SRF, CMT, and SFCT between baseline and 1-month follow-up were performed using the Student paired $t$-test. In all analyses, $p$ values $<0.05$ were considered statistically significant.

\section{Results}

A total of 30 eyes of 26 Caucasian patients (4 females and 22 males) were included in the study: 17 eyes of 15 patients were included in the PDT group, while 13 eyes of 11 patients were included in the aflibercept group. Patients included in the two groups were homogeneous for age (mean age $53 \pm 11$ years and $58 \pm 8$ years, respectively $[p=$ $0.196]$ ) and sex (2 females and 13 males in PDT group, 2 females and 9 males in aflibercept group $[p=0.906])$. Considering all 30 included eyes, 18 (60\%) were treatmentnaïve (10 in PDT group and 8 in aflibercept group), while
12 (40\%) (7 in PDT group and 5 in aflibercept group) were previously treated with aflibercept injections (5 patients), half-fluence PDT (3 patients), or both treatments (4 patients). Of note, according to exclusion criteria, all previous treatments were performed at least 6 months before the inclusion data. The CNVs complicating chronic CSC were in all cases type 1 neovascularizations [7].

At the baseline, no significant difference was disclosed between the two groups considering BCVA, structural OCT parameters (CMT, SRF, and SFCT) and OCT-A parameters (CNV area and VD) (Table 1). Furthermore, no significant correlation was seen between BCVA and each structural OCT and OCT-A parameters ( $p>0.3$ in all analyses).

\section{Clinical and structural OCT changes after treatment}

Considering the PDT group, mean BCVA improved from $0.24 \pm 0.20$ LogMAR (range 0.0-0.7 LogMAR; approximately between $20 / 32$ and 20/40 Snellen equivalent) to $0.18 \pm 0.16$ LogMAR (range 0.0-0.5 LogMAR; 20/32 Snellen equivalent) after the treatment, although this difference was not statistically significant $(p=0.086)$. In the aflibercept group, BCVA significantly improved after the treatment, from $0.35 \pm 0.23$ LogMAR (range 0.1-1.0 LogMAR; approximately between 20/40 and 20/50 Snellen equivalent) to $0.24 \pm 0.15 \mathrm{LogMAR}$ (range $0.0-0.5 \mathrm{Log}$ MAR; approximately between 20/32 and 20/40 Snellen equivalent) $(p=0.024)$ (Table 2). No difference in BCVA at baseline and at 1-month follow-up was disclosed between PDT and aflibercept group $(p=0.196$ and $p=0.328$, respectively).

Both treatments achieved an excellent anatomical response: CMT and SRF significantly decreased in both groups. In detail, CMT significantly decreased from $337 \pm$ $77 \mu \mathrm{m}$ (range $224-499 \mu \mathrm{m}$ ) to $265 \pm 65 \mu \mathrm{m}$ (range 165-394 $\mu \mathrm{m})(p=0.005)$ in the PDT group, and from $307 \pm 75 \mu \mathrm{m}$ (range $220-443 \mu \mathrm{m}$ ) to $276 \pm 52 \mu \mathrm{m}$ (range $210-363 \mu \mathrm{m}$ ) $(p=0.041)$ in the aflibercept group. In the same way, SRF significantly decreased from $121 \pm 57 \mu \mathrm{m}$ (range 57-242 $\mu \mathrm{m})$ to $58 \pm 58 \mu \mathrm{m}($ range $0-169 \mu \mathrm{m})(p=0.011)$ in the PDT group, and from $109 \pm 55 \mu \mathrm{m}$ (range $25-213 \mu \mathrm{m}$ ) to $65 \pm 47 \mu \mathrm{m}$ (range $0-173 \mu \mathrm{m})(p=0.025)$ in the aflibercept group (Table 2). Also in this analysis, no difference in CMT and SRF at 1 month was disclosed between two groups ( $p=0.652$ and $p=0.730$, respectively).

At the SFCT analysis, a significant reduction was appreciated in both PDT group and aflibercept group after the treatment: from $396 \pm 96 \mu \mathrm{m}$ (range $200-550 \mu \mathrm{m}$ ) to $362 \pm 106 \mu \mathrm{m} \quad$ (range $112-541 \mu \mathrm{m}) \quad(p=0.003)$ in the PDT group, and from $359 \pm 108 \mu \mathrm{m}$ (range $241-558 \mu \mathrm{m}$ ) to $319 \pm 109 \mu \mathrm{m}$ (range $181-534 \mu \mathrm{m})(p=0.014)$ in the aflibercept group (Table 2). No difference in SFCT at 1 month was disclosed between two groups $(p=0.283)$. 


\section{CNV changes at OCT-A after treatment}

Using OCT-A, CNVs were well visualized in all patients. At baseline, CNVs typically appeared as large vessels with several vascular anastomotic connections and a paucity of capillaries within the lesions. Interestingly, the treatment seemed to produce minimal qualitative changes to the CNVs in both groups (Figs. 1-3).

Considering the quantitative evaluation, mean $\mathrm{CNV}$ area slightly decreased from the baseline to the 1-month follow-up (from $0.586 \pm 0.449 \mathrm{~mm}^{2}$ to $0.553 \pm 0.453 \mathrm{~mm}^{2}$ and from $0.767 \pm 0.466 \mathrm{~mm}^{2}$ to $0.733 \pm 0.472 \mathrm{~mm}^{2}$, in PDT and aflibercept group, respectively). However, these results were of doubtful significance, because the decrease was statistically significance only in PDT group $(p=$ $0.041)$ and not in aflibercept group $(p=0.095)$ (Table 2). Of note, no difference in CNV area at both baseline and after the treatment was observed between the two groups ( $p=0.227$ and 0.721 , respectively). Considering only the 18 treatment-naïve eyes, the same results were obtained (from $0.664 \pm 0.510 \mathrm{~mm}^{2}$ to $0.658 \pm 0.527 \mathrm{~mm}^{2}$ $[p=0.637]$ and from $0.825 \pm 0.481 \mathrm{~mm}^{2}$ to $0.793 \pm$ $0.483 \mathrm{~mm}^{2}[p=0.185]$, in PDT and aflibercept group, respectively) (Figs. 1 and 2). Interobserver variability between the two readers was excellent for $\mathrm{CNV}$ area measurements $(\mathrm{ICC}=0.953[0.905-0.978]$ ).

VD evaluation between baseline and the 1-month followup turned out to be not statistically significant in both groups. In fact, VD changed from $0.444 \pm 0.097$ to $0.434 \pm 0.073(p=0.502)$ in PDT group, and from $0.406 \pm$ 0.058 to $0.425 \pm 0.071(p=0.086)$ in aflibercept group (Figs. 1-3). Of note, also in this case, the same results were achieved considering only 18 treatment-naïve eyes (from $0.455 \pm 0.091$ to $0.442 \pm 0.083[p=0.346]$ and from $0.420 \pm 0.060$ to $0.426 \pm 0.066[p=0.140]$, in PDT and aflibercept group, respectively).

\section{Discussion}

In this study, CNVs complicating chronic CSC share common early (1 month) vascular changes at OCT-A after half-fluence PDT or aflibercept intravitreal injection in terms of VD. In fact, in both groups, VD did not show any significant change. However, the $\mathrm{CNV}$ area decreased significantly only in the PDT group (and only a trend was appreciated in the aflibercept group). While these results are of doubtful significance (the different sample size of the sample of the two groups must also be taken into account), one may hypothesize that, after a single treatment administration, PDT has a stronger effect than aflibercept on CNV shrinking but, similarly to aflibercept, no effects on VD (i.e., on the arteriogenesis).
Pachychoroid neovasculopathy is a recently described clinical entity that refers to type 1 neovascularization in patients with choroidal thickening and/or choroidal dilated vessels in absence of AMD signs [7]. Type 1 neovascularization complicating CSC is typically localized in a flat irregular PED that is frequently observed in the chronic form of CSC [1, 17, 19, 20]. However, several authors studied the prevalence of $\mathrm{CNV}$ contained in flat irregular PEDs in CSC with variable results. Dansingani et al. [8] reported that a shallow irregular PED on structural OCT was associated with a type 1 neovascularization in 21 out of 22 cases (95\%). On the other hand, Bousquet et al. [17] analyzing 88 chronic CSC patients with flat irregular PED found that OCT-A detected the presence of a type $1 \mathrm{CNV}$ in only $35.6 \%$ of cases. Despite the great variability of the results, all authors agreed that OCT-A detected CNV more frequently than any other imaging modalities, including dye angiographies [8, 16-18]. In fact, in the analysis of CNV features, OCT-A overcomes some important limitations of traditional dye based angiography techniques, including the absence of leakage effect and the capability to independently segment the retinal and choroidal vascular plexuses.

Although the OCT-A imaging changes of CNVs after anti-VEGF treatment have been previously well described in the setting of AMD [10, 21-24], to the best of our knowledge, no studies have been published about changes of type 1 neovascularization complicating the chronic form of CSC after treatment using OCT-A. In the setting of AMD, Lumbroso et al. [21] demonstrated an immediately VD and CNV area decrease of type $2 \mathrm{CNV}$ after anti-VEGF injections. This was due to the loss of smaller capillaries after each injection followed by a partial reopening, even if the CNV average area and VD remains smaller [21]. Also, in type $1 \mathrm{CNV}$-complicating AMD, a consistent size reduction in CNV area after anti-VEGF treatment was described [23, 24]. However, Spaide [22] reported that type $1 \mathrm{CNV}$ receiving recurrent periodic antiangiogenic treatment showed the preservation of central trunk vessels, that appeared large even in small lesions with many vascular anastomotic connections among larger vessels and a paucity of capillaries visualized within the lesions.

In case of CNV complicating chronic CSC, we demonstrated that CNVs showed less changes 1 month after treatment in comparison to AMD. In fact, in both PDT and aflibercept groups, the VD did not show any significant change (Figs. 1-3), although a slightly reduction in CNV area was observed and all patients showed a favorable clinical response. Furthermore, also the CNV appearance was different in comparison with AMD because, in our series, CNVs were typically characterized by large-caliber vessels with a paucity of capillaries within the lesions even at the baseline. These different structural features between chronic CSC and AMD neovascularization, could be 
Fig. 1 Multimodal imaging evaluation of a previous treated patient affected by choroidal neovascularization (CNV) complicating chronic central serous chorioretinopathy before and 1 month after treatment (half-fluence photodynamic therapy). First row: Multicolor imaging, short-wave fundus autofluorescence, and infrared reflectance at the baseline. Left panel: Horizontal structural optical coherence tomography (OCT) passing through the fovea, magnified $3 \times 3$ enface OCT-angiography (OCT-A) image before and after the binarization, and corresponding $\mathrm{B}$-scans showing the presence of a neovascular network at the baseline (CNV boundary colored with yellow-dashed lines). Right panel: Horizontal structural OCT passing through the fovea, magnified $3 \times 3$ enface OCT-A image before and after the binarization, and corresponding B-scans showing the presence of a neovascular network after the treatment (CNV boundary colored with yellow-dashed lines). Structural OCT disclosed the complete reabsorption of subretinal fluid. OCT-A images illustrated a slightly reduction in $\mathrm{CNV}$ area and no significant modifications in the vessel density
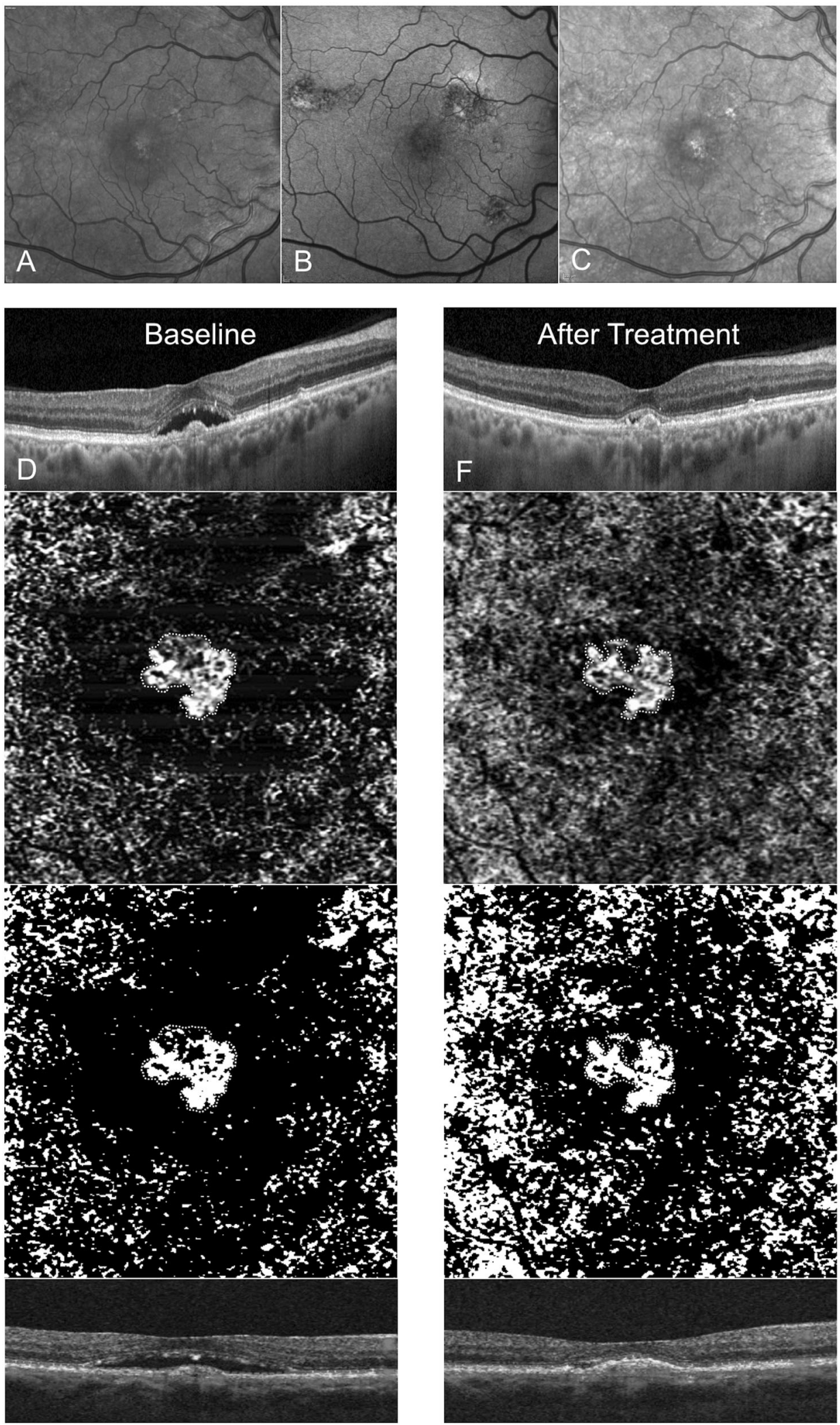

explained by the different genesis of the two diseases. Even if the pathogenesis of CNV complicating CSC is still partly unknown, Fung et al. [25] speculated that long-standing
PED characterizing chronic CSC could result into Bruch's membrane disruption and then stimulate the development of neovascularization. Contrary to what happens in AMD, this 
Fig. 2 Multimodal imaging evaluation of a treatment-naïve patient affected by choroidal neovascularization (CNV) complicating chronic central serous chorioretinopathy before and 1 month after treatment (1 aflibercept $2.0 \mathrm{mg} / 0.05 \mathrm{ml}$ intravitreal injection). First row: Multicolor imaging, short-wave fundus autofluorescence, and infrared reflectance at the baseline. Left panel: Horizontal structural optical coherence tomography (OCT) passing through the fovea, magnified $3 \times 3$ enface OCT-angiography (OCT-A) image before and after the binarization, and corresponding B-scans showing the presence of a neovascular network at the baseline (CNV boundary colored with yellowdashed lines). Right panel: Horizontal structural OCT passing through the fovea, magnified $3 \times 3$ enface OCT-A image before and after the binarization, and corresponding $\mathrm{B}$-scans showing the presence of a neovascular network after the treatment $(\mathrm{CNV}$ boundary colored with yellow-dashed lines). Structural OCT disclosed the complete reabsorption of subretinal fluid. OCT-A images illustrated no significant modifications in CNV area and vessel density
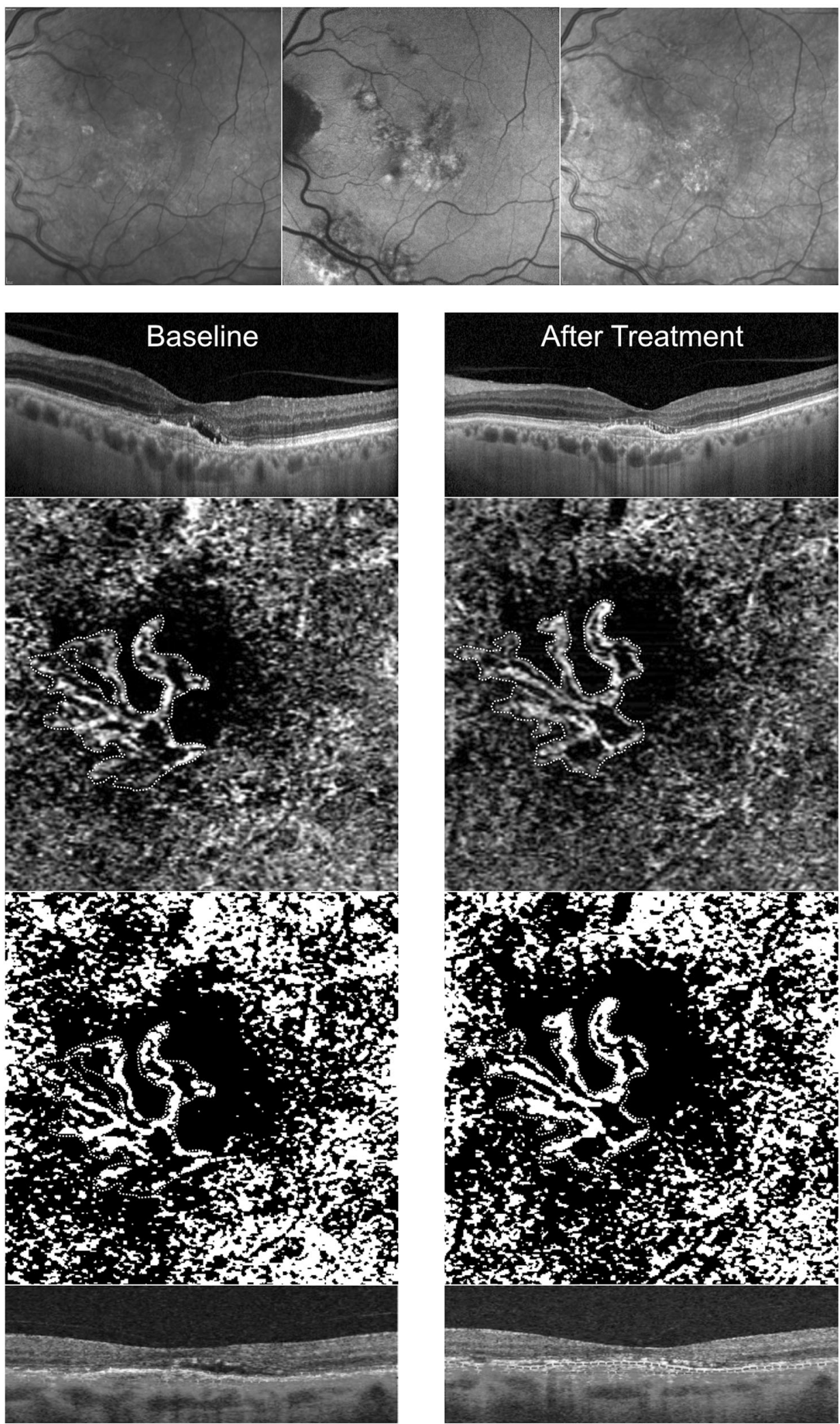

process would be characterized by a long-standing growth of neovessels that could play a "compensatory" role in the chronic CSC. In fact, SRF in cases of flat vascularized PED might not always be a sign of CNV activity [17]. 
Fig. 3 Multimodal imaging evaluation of a treatment-naïve patient affected by choroidal neovascularization (CNV) complicating chronic central serous chorioretinopathy before and 1 month after treatment (half-fluence photodynamic therapy). First row: infrared reflectance, short-wave fundus autofluorescence, and nearinfrared fundus autofluorescence at the baseline. Left panel: Horizontal structural optical coherence tomography (OCT) passing through the fovea, magnified $3 \times 3$ enface OCTangiography (OCT-A) image before and after the binarization, and corresponding B-scans showing the presence of a neovascular network at the baseline (CNV boundary colored with yellow-dashed lines). Right panel: Horizontal structural OCT passing through the fovea, magnified $3 \times 3$ enface OCT-A image before and after the binarization, and corresponding B-scans showing the presence of a neovascular network after the treatment (CNV boundary colored with yellow-dashed lines). Structural OCT disclosed the persistence of subretinal fluid after the treatment. OCT-A images illustrated a slightly reduction in $\mathrm{CNV}$ area and no significant modifications in the vessel density
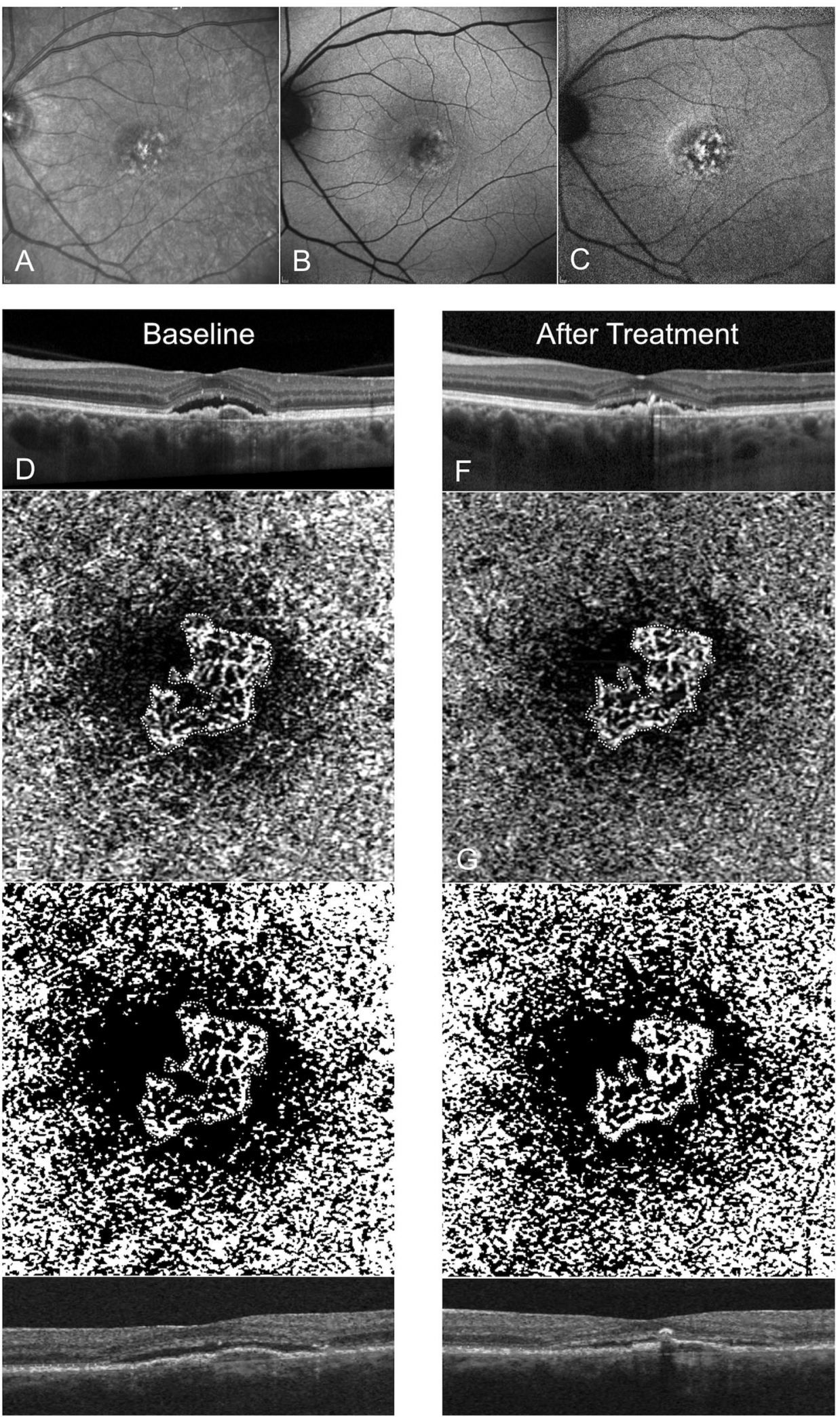
Interestingly, Spaide [22] speculated that, in the CNV genesis, two different mechanisms were implicated: the angiogenesis, that is characterized by new-vessels proliferation and high VEGF dependence, and the arteriogenesis, that is characterized by the dilation of preexisting channels and not high-VEGF dependence. We hypothesized that the different imaging aspect and modification after the treatment of CNVs between AMD patients (well reported in the literature) and chronic CSC patients (reported firstly in our series) could be due to the different ratio between angiogenesis and arteriogenesis. Of note, we believe that the expansion of the trunk and large branches (arteriogenesis) is the main driving force of CNV in CSC, whereas the growth and branching of vascular sprouts (angiogenesis) is the main driving force of $\mathrm{CNV}$ in AMD. This theory would also explain the lack or reduction in $\mathrm{CNV}$ area of the aflibercept group; indeed, the arteriogenesis, the main force driving the development of $\mathrm{CNV}$ in this kind of patients, is not a VEGF dependent process [22]. The different genesis of CNV in these two diseases (CSC and AMD) was also supported by the different ICGA features: the choroidal vascular plaque displayable in the late phases of ICGA is a peculiar finding of type 1 neovascularization in AMD but it is visualized in only $25-42 \%$ of cases in type 1 neovascularization complicating CSC [8-11]. Moreover, our group described that the neovascular network in pachychoroid eyes visualized by OCT-A often correspond to the late hypofluorescent area at ICGA, in turn a wash-out effect, supporting again the dilation of $\mathrm{CNV}$ vessels (arteriogenesis) [11-18].

Finally, our data confirmed the effect of both half-fluence PDT and aflibercept injection in the reduction of SFCT. If the effect of the PDT on SFCT has already been well described in the literature [26-28], on the other hand, the reduction in the SFCT after aflibercept injections in patients with pachychoroid neovasculopathy was only recently reported [29]. As supposed by Padrón-Pérez et al. [29], we hypothesized that the reduction of SFCT might be attributable to a reduction in choroidal vascular permeability secondary to the aflibercept injections.

We acknowledge that this study has several limitations, mainly due to the retrospective nature, the relatively small sample size and short follow-up. Lastly, although we cannot completely rule out that previous treatment partially influenced the findings of CNV evaluation at OCT-A, despite we excluded patients with previous treatments in the last 6 months, it is noteworthy that our results were confirmed in the subanalysis of treatment-naïve eyes (60\% of all eyes).

In conclusion, we demonstrated that, despite all patients showed a favorable clinical response, VD of CNVs complicating chronic CSC did not change after treatment (both halffluence PDT and aflibercept intravitreal injection). These findings seem to support arteriogenesis as the main driving force of $\mathrm{CNV}$ in pachychoroid-related macular disorders.
However, further studies with larger number of patients and analyzing CNV during recurrent periodic treatments should be conducted to confirm our preliminary findings.

\section{Summary}

\section{What was known before}

- Choroidal neovascularization (CNV) could complicate chronic central serous chorioretinopathy (CSC).

\section{What this study adds}

- We analyzed CNV features using optical coherence tomography angiography in chronic CSC before and after the treatment. We demonstrated that vessel density of CNVs did not change after treatment.

Acknowledgements Financial disclosures: RS, LT, EC, AC, LQ, SC: none. FB is a consultant for Alcon (Fort Worth,Texas,USA), Alimera Sciences (Alpharetta, Georgia, USA), Allergan Inc (Irvine, California, USA), Farmila-Thea (Clermont-Ferrand, France), Bayer SheringPharma (Berlin, Germany), Bausch and Lomb (Rochester, New York, USA), Genentech (San Francisco, California, USA), HoffmannLa-Roche (Basel, Switzerland), Novagali Pharma (Évry, France), Novartis(Basel, Switzerland), Sanofi-Aventis (Paris, France), Thrombogenics (Heverlee,Belgium), and Zeiss (Dublin, USA). GQ is a consultant for Amgen (Thousand Oaks, California, USA), Alimera Sciences (Alpharetta, Georgia, USA), Allergan Inc (Irvine, California, USA), Bayer Shering-Pharma (Berlin, Germany), Bausch And Lomb (Rochester, New York, USA), Heidelberg (Germany), KHB (Shanghai, China), Novartis (Basel, Switzerland), Roche (Basel, Switzerland), Sandoz (Berlin, Germany), and Zeiss (Dublin, USA).

\section{Compliance with ethical standards}

Conflict of interest The authors declare that they have no conflict of interest.

Publisher's note: Springer Nature remains neutral with regard to jurisdictional claims in published maps and institutional affiliations.

\section{References}

1. Warrow DJ, Hoang QV, Freund KB. Pachychoroid pigment epitheliopathy. Retina 2013;33:1659-72.

2. Kitzmann AS, Pulido JS, Diehl NN, Hodge DO, Burke JP. The incidence of central serous chorioretinopathy in Olmsted County, Minnesota,1980-2002. Ophthalmology 2008;115:169-73.

3. Liew G, Quin G, Gillies M, et al. Central serous chorioretinopathy: a review of epidemiology and pathophysiology. Clin Exp Ophthalmol. 2013;41:201-14.

4. Wang M, Munch IC, Hasler PW, Prunte C, Larsen M. Central serous chorioretinopathy. Acta Ophthalmol. 2008;86:126-45. 
5. Quin G, Liew G, Ho IV, Gillies M, Fraser-Bell S. Diagnosis and interventions for central serous chorioretinopathy: review and update. Clin Exp Ophthalmol. 2013;41:187-200.

6. Loo RH, Scott IU, Flynn HW Jr, et al. Factors associated with reduced visual acuity during long-term follow-up of patients with idiopathic central serous chorioretinopathy. Retina 2002;22:19-24.

7. Pang CE, Freund KB. Pachychoroid neovasculopathy. Retina 2015;35:1-9.

8. Dansingani KK, Balaratnasingam C, Klufas MA, Sarraf D, Freund $\mathrm{KB}$. Optical coherence tomography angiography of shallow irregular pigment epithelial detachments in pachychoroid spectrum disease. Am J Ophthalmol. 2015;160:1243-.e2.

9. Carnevali A, Cicinelli MV, Capuano V, et al. Optical coherence tomography angiography: a useful tool for diagnosis of treatmentnaïve quiescent choroidal neovascularization. Am J Ophthalmol. 2016;169:189-98.

10. Kuehlewein L, Bansal M, Lenis TL, et al. Optical coherence tomography angiography of type 1 neovascularization in age-related macular degeneration. Am J Ophthalmol. 2015;160:739-48.

11. Sacconi R, Baldin G, Carnevali A, et al. Response of central serous chorioretinopathy evaluated by multimodal retinal imaging. Eye 2018;32:734-42.

12. Sacconi R, Freund KB, Yannuzzi LA, et al. The expanded spectrum of perifoveal exudative vascular anomalous complex. Am J Ophthalmol. 2017;184:137-46.

13. Sacconi R, Corbelli E, Carnevali A, Querques L, Bandello F, Querques G. Optical coherence tomography angiography in geographic atrophy. Retina 2018;38:2350-5.

14. Carnevali A, Sacconi R, Corbelli E, et al. Optical coherence tomography angiography analysis of retinal vascular plexuses and choriocapillaris in patients with type 1 diabetes without diabetic retinopathy. Acta Diabetol. 2017;54:695-702.

15. Matet A, Daruich A, Dirani A, Ambresin A, Behar-Cohen F. Macular telangiectasia type 1: capillary density and microvascular abnormalities assessed by optical coherence tomography angiography. Am J Ophthalmol. 2016;167:18-30.

16. Demirel S, Yanık Ö, Nalcı H, Batıoglu F, Özmert E. The use of optical coherence tomography angiography in pachychoroid spectrum diseases: a concurrent comparison with dye angiography. Graefes Arch Clin Exp Ophthalmol. 2017;255:2317-24.

17. Bousquet E, Bonnin S, Mrejen S, Krivosic V, Tadayoni R, Gaudric A. Optical coherence tomography angiography of flat irregular pigment epithelium detachment in chronic central serous chorioretinopathy. Retina. 2018;38:629-38.

18. Carnevali A, Capuano V, Sacconi R, et al. Optical coherence tomography angiography of treatment-naïve quiescent choroidal neovascularization in pachychoroid neovasculopathy. Ophthalmol Retin. 2017;1:328-32.

19. Daruich A, Matet A, Dirani A, et al. Central serous chorioretinopathy: recent findings and new physiopathology hypothesis. Prog Retin Eye Res. 2015;48:82-118.

20. Song IS, Shin YU, Lee BR. Time-periodic characteristics in the morphology of idiopathic central serous chorioretinopathy evaluated by volume scan using spectral-domain optical coherence tomography. Am J Ophthalmol. 2012;154:366-e4.

21. Lumbroso B, Rispoli M, Savastano MC. Longitudinal optical coherence tomography-angiography study of type 2 naive choroidal neovascularization early response after treatment. Retina 2015;35:2242-51.

22. Spaide RF. Optical coherence tomography angiography signs of vascular abnormalization with antiangiogenic therapy for choroidal neovascularization. Am J Ophthalmol. 2015;160: $6-16$.

23. De Carlo TE, Bonini Filho MA, Chin AT, et al. Spectral- domain optical coherence tomography angiography of choroidal neovascularization. Ophthalmology 2015;122:1228-38.

24. Muakkassa NW, Chin AT, de Carlo T, et al. Characterizing the effect of anti-vascular endothelial growth factor therapy on treatment-naive choroidal neovascularization using optical coherence tomography angiography. Retina 2015;35:2252-9.

25. Fung AT, Yannuzzi LA, Freund KB. Type 1 (sub-retinal pigment epithelial) neovascularization in central serous chorioretinopathy masquerading as neovascular age-related macular degeneration. Retina 2012;32:1829-37.

26. Demirel S, Özcan G, Yanık Ö, Batıŏ̆lu F, Özmert E. Vascular and structural alterations of the choroid evaluated by optical coherence tomography angiography and optical coherence tomography after half-fluence photodynamic therapy in chronic central serous chorioretinopathy. Graefes Arch Clin Exp Ophthalmol. 2019 https://doi.org/10.1007/s00417-018-04226-6.

27. Ma DJ, Park UC, Kim ET, Yu HG. Choroidal vascularity changes in idiopathic central serous chorioretinopathy after half-fluence photodynamic therapy. PLoS ONE. 2018;13:e0202930.

28. van Dijk EHC, Fauser S, Breukink MB, et al. Half-dose photodynamic therapy versus high-density subthreshold micropulse laser treatment in patients with chronic central serous chorioretinopathy: the PLACE trial. Ophthalmology 2018;125: 1547-55.

29. Padrón-Pérez N, Arias L, Rubio M, et al. Changes in choroidal thickness after intravitreal injection of anti-vascular endothelial growth factor in pachychoroid neovasculopathy. Invest Ophthalmol Vis Sci. 2018;59:1119-24. 